\title{
A semilinear elliptic equation with competing powers and a radial potential
}

\author{
Monica Musso and Juliana Pimentel
}

June 15, 2021

\begin{abstract}
We verify the existence of radial positive solutions for the semi-linear equation

$$
-\Delta u=u^{p}-V(y) u^{q}, \quad u>0, \quad \text { in } \mathbb{R}^{N}
$$

where $N \geq 3, p$ is close to $p^{*}:=(N+2) /(N-2)$, and $V$ is a radial smooth potential. If $q$ is super-critical, namely $q>p^{*}$, we prove that this Problem has a radial solution behaving like a super-position of bubbles blowing-up at the origin with different rates of concentration, provided $V(0)<0$. On the other hand, if $N /(N-2)<q<p^{*}$, we prove that this Problem has a radial solution behaving like a super-position of flat bubbles with different rates of concentration, provided $\lim _{r \rightarrow \infty} V(r)<0$.
\end{abstract}

\section{Introduction}

Let $N \geq 3$ and consider

$$
-\Delta u=u^{p}-V(y) u^{q}, \quad u>0, \quad \text { in } \mathbb{R}^{N}
$$

where $V \in L^{\infty}\left(\mathbb{R}^{N}\right), N \geq 3, q>p^{s}, p>p^{*}$, with

$$
p^{s}=\frac{N}{N-2}, \quad p^{*}=\frac{N+2}{N-2} .
$$

In this paper, we are interested to the case $p$ slightly super-critical,

$$
\left\{\begin{array}{l}
-\Delta u=u^{p^{*}+\epsilon}-V(y) u^{q}, \text { in } \mathbb{R}^{N} \\
u(y) \rightarrow 0, \text { as }|y| \rightarrow \infty
\end{array}\right.
$$


where $\epsilon>0$.

For $q=1$, problem (2) was treated in the critical case $(\epsilon=0)$ in [3] and in the sub-critical case $(\epsilon<0)$ in [8] . The super-critical analogue $(\epsilon>0)$ was addressed in [11], where it was proved the existence of a radial positive solution to (2) when $V$ is a radial smooth function with $V(0)<0$. A previous construction can also be found in [12].

In [2], the authors consider problem (2) for any fixed $q$ satisfying $p^{s}<q<p^{*}$. It was proved the existence of an increasing number of rapidly decaying ground states, that is, solutions $u$ of (2) such that $\lim _{|x| \rightarrow \infty} u(x)=0$. The result in [2] is obtained via tools in geometrical dynamical systems. The same equation was also treated in [10] and in [7], using a different approach, which also provided precise asymptotics for the solutions. In bounded domains, the class of radial solutions behaving like a super-position of spikes was treated in the setting of super-critical exponents, in [13, 14].

Let us now consider problem (2) in the super-critical case $(\epsilon>0)$. In the case of a single power, i.e., $p^{*}+\epsilon=q$, and when $V(y) \equiv 1$, equation (2) is equivalent to

$$
\left\{\begin{array}{l}
\Delta u+u^{p^{*}}=0, \text { in } \mathbb{R}^{N} \\
u>0, \text { in } \mathbb{R}^{N}
\end{array}\right.
$$

if we let $\epsilon$ goes to zero. It is well known that all bounded solutions of (8) are of the form

$$
w_{\lambda, \xi}(x)=\gamma_{N}\left(\frac{\lambda}{\lambda^{2}+|y-\xi|^{2}}\right)^{\frac{N-2}{2}}, \quad \gamma_{N}=(N(N-2))^{\frac{N-2}{4}}
$$

where $\lambda$ is a positive parameter and $\xi \in \mathbb{R}^{N},[1,16,4]$. These functions are known in the literature as bubbles.

We want to prove the existence of a solution whose shape resembles a superposition of bubbles around the origin 0 with different blow-up orders. This class of concentration phenomena is known as bubble-tower. In the setting of semilinear elliptic equations with radial symmetry, these solutions were detected in a few situations, as we can see for instance in [13, 5, 6, 11, 10].

Bubble-towers highly concentrated around the origin exist for (2) under the assumption that and $V(0)<0$. This is the content of our first result.

Theorem 1.1. Let $N \geq 3$ and $p^{s}<q<p^{*}<p$. Assume that $V \in L^{\infty}\left(\mathbb{R}^{N}\right)$ and $V(0)<0$. Then for every integer $k \geq 1$ there exists $\epsilon_{k}>0$ such that, for any 
$\epsilon \in\left(0, \epsilon_{k}\right)$, a solution $u_{\epsilon}$ of (2) exists and it has the form

$$
u_{\epsilon}(x)=\gamma_{N} \sum_{j=1}^{k}\left(\frac{1}{1+\alpha_{j^{\frac{4}{N-2}}} \epsilon^{-\left(j-1+\frac{1}{p^{*}-q}\right)^{\frac{4}{N-2}}}|x|^{2}}\right)^{\frac{N-2}{2}} \alpha_{j} \epsilon^{-\left(j-1+\frac{1}{p^{*}-q}\right)}(1+o(1))
$$

with o(1) $\rightarrow 0$ uniformly on compact sets of $\mathbb{R}^{N}$, as $\epsilon \rightarrow 0$. The constants $\alpha_{i}$ have explicit expression and depend only on $k, N, q$ and $V(0)$,

$$
\alpha_{j}=\left[-\frac{a_{5} V(0)\left(p^{*}-q\right)}{a_{3} k}\right]^{\frac{1}{p^{*}-q}}\left(\frac{a_{2}}{a_{3}}\right)^{j-1} \frac{(k-j) !}{(k-1) !} \quad j=1, \cdots, k,
$$

while $a_{2}, a_{3}, a_{5}$ are the positive constants defined in (19).

Also in the case in which $p^{s}<p^{*}<p<q$ bubble-towers do exist, but they are of a different nature, and their existence depends on the behavior of the potential $V$ at infinity.

Theorem 1.2. Let $N \geq 3$ and $q>p^{*}$. Assume that $V \in L^{\infty}\left(\mathbb{R}^{N}\right)$ and $V_{\infty}:=$ $\lim _{|x| \rightarrow \infty} V(x)<0$. Then for every integer $k \geq 1$ there exists $\epsilon_{k}>0$ such that, for any $\epsilon \in\left(0, \epsilon_{k}\right)$, a solution $u_{\epsilon}$ of (2) exists and it has the form

$$
\hat{u}_{\epsilon}(x)=\gamma_{N} \sum_{j=1}^{k}\left(\frac{1}{1+\hat{\alpha}_{j}^{\frac{4}{N-2}} \epsilon^{\left(j-1+\frac{1}{q-p^{*}}\right) \frac{4}{N-2}}|x|^{2}}\right)^{\frac{N-2}{2}} \hat{\alpha}_{j} \epsilon^{\left(j-1+\frac{1}{q-p^{*}}\right)}(1+o(1))
$$

with o(1) $\rightarrow 0$ uniformly on compact sets of $\mathbb{R}^{N}$, as $\epsilon \rightarrow 0$. The constants $\hat{\alpha}_{i}$ have explicit expression and depend only on $k, N, q$ and $V_{\infty}$,

$$
\hat{\alpha}_{j}=\left[\frac{\hat{a}_{5} V_{\infty}\left(p^{*}-q\right)}{a_{3} k}\right]^{\frac{1}{p^{*}-q}}\left(\frac{a_{2}}{a_{3}}\right)^{j-1} \frac{(k-j) !}{(k-1) !}, \quad j=1, \cdots, k,
$$

while $a_{2}, a_{3}, \hat{a}_{5}$ are the positive constants defined in (19) and (37).

The bubble-tower in (6) describes a superposition of $k$ flat bubbles.

In order to prove our results, we start by reducing the problem to a nonautonomous ordinary differential equation, using the so-called Emden-Fowler transformation, [9]. Then we perform a Lyapunov-Schmidt reduction, as in [15], to reduce the procedure of construction of solutions to a finite-dimensional variational problem. 
The paper is organized as follows. In Section 2, we provide an asymptotic expansion of the energy functional associated to the ODE problem. The finite dimensional reduction argument is discussed in Section 3. We prove Theorems 1.1 and 1.2 in Sections 4 and 5, respectively.

\section{The energy asymptotic expansion}

Since we are seeking for a solution $u$ of (2) with fast decay, we can assume that $u$ is radial around the origin. Then we arrive at the following equivalent problem

$$
\left\{\begin{array}{l}
u^{\prime \prime}(r)+\frac{N-1}{r} u^{\prime}(r)+u^{p^{*}+\epsilon}(r)-V(r) u^{q}(r)=0 \\
u(r) \rightarrow 0, \text { as } r \rightarrow \infty
\end{array}\right.
$$

By introducing the so-called Emden-Fowler transformation,

$$
v(x)=r^{\frac{2}{p^{*}-1}} u(r), \quad \text { with } r=e^{-\frac{p^{*}-1}{2} x},
$$

for $x \in \mathbb{R}$, the problem (8) becomes

$$
\left\{\begin{array}{l}
v^{\prime \prime}(x)-v(x)+\beta\left[e^{\epsilon x} v^{p^{*}+\epsilon}(x)-V\left(e^{-\frac{p^{*}-1}{2} x}\right) e^{-\left(p^{*}-q\right) x} v^{q}(x)\right]=0 \\
0<v(x) \rightarrow 0, \text { as }|x| \rightarrow \infty
\end{array}\right.
$$

in $\mathbb{R}$, where $\beta=\left(\frac{2}{N-2}\right)^{2}$. We henceforth denote $\omega(x)=V\left(e^{-\frac{p^{*}-1}{2} x}\right)$.

The energy functional related to (10) is

$$
E_{\epsilon}(\psi)=I_{\epsilon}(\psi)+\frac{\beta}{q+1} \int_{\mathbb{R}} \omega(x) e^{-\left(p^{*}-q\right) x}|\psi|^{q+1} d x
$$

where

$$
I_{\epsilon}(\psi)=\frac{1}{2} \int_{\mathbb{R}}\left(\left|\psi^{\prime}\right|^{2}+|\psi|^{2}\right) d x-\frac{\beta}{p^{*}+\epsilon+1} \int_{\mathbb{R}} e^{\epsilon x}|\psi|^{p^{*}+\epsilon+1} d x .
$$

Let us consider the positive radial solution of

$$
\Delta w+w^{p^{*}}=0, \quad w(0)=\gamma_{N}
$$

given by $w(r)=\gamma_{N}\left(\frac{1}{1+r^{2}}\right)^{\frac{N-2}{2}}$. Now we set $U$ to be the Emden-Fowler transformation of $w$

$$
U(x)=\gamma_{N} e^{-x}\left(1+e^{-\left(p^{*}-1\right) x}\right)^{-\frac{N-2}{2}} .
$$


Then $U$ satisfies

$$
U^{\prime \prime}-U+\beta U^{p^{*}}=0, \quad 0<U(x) \rightarrow 0, \text { as }|x| \rightarrow \infty
$$

It is then natural to look for a solution of (10) of the form

$$
v(x)=\sum_{i=1}^{k} U\left(x-\xi_{i}\right)+\phi(x)
$$

for certain choice of points $0<\xi_{1}<\xi_{2}<\cdots<\xi_{k}$ and $\phi$ is small. We set

$$
U_{i}(x)=U\left(x-\xi_{i}\right), \quad \bar{U}=\sum_{i=1}^{k} U_{i}(x)
$$

and choose the points $\xi_{i}$ as follows:

$$
\begin{gathered}
\xi_{1}=-\frac{1}{p^{*}-q} \log \epsilon-\log \Lambda_{1} \\
\xi_{i+1}-\xi_{i}=-\log \epsilon-\log \Lambda_{i+1}, \quad i=1, \cdots, k-1
\end{gathered}
$$

where the $\Lambda_{i}$ 's are positive parameters. This choice of the $\xi_{i}$ 's turns out to be convenient in the proof of the following asymptotic expansion of $E_{\epsilon}(\bar{U})$. We set $\Lambda=\left(\Lambda_{1}, \Lambda_{2}, \cdots, \Lambda_{k}\right)$.

Lemma 2.1. Let $N \geq 3, \delta>0$ fixed, $k \in \mathbb{N}$. Assume that

$$
\delta<\Lambda_{i}<\delta^{-1}, \quad i=1,2, \cdots, k .
$$

Then there exist positive numbers $a_{1}, i=1, \cdots, 5$, depending on $N, p$ and $q$, such that

$$
E_{\epsilon}(\bar{U})=k a_{1}+\epsilon \Psi_{k}(\Lambda)+k \epsilon \beta a_{4}+\epsilon \theta_{\epsilon}(\Lambda)-\frac{a_{3} k}{2\left(p^{*}-p\right)}\left((1-k)\left(p^{*}-q\right)-2\right) \epsilon \log \epsilon
$$

where

$$
\Psi_{k}(\Lambda)=a_{3} k \log \Lambda_{1}+a_{5} V(0) \Lambda_{1}^{\left(p^{*}-q\right)}+\sum_{i=1}^{k}\left[(k-i+1) a_{3} \log \Lambda_{i}-a_{2} \Lambda_{i}\right]
$$

and $\theta_{\epsilon}(\Lambda) \rightarrow 0$ as $\epsilon \rightarrow 0$ uniformly in $C^{1}$-sense on the set of $\Lambda_{i}$ 's satisfying (17). 
Proof. We estimate

$$
\begin{aligned}
I_{\epsilon}(\bar{U})= & \frac{1}{2} \int_{\mathbb{R}}\left(\left|\overline{U^{\prime}}\right|^{2}+|\bar{U}|^{2}\right) d x-\frac{\beta}{p^{*}+\epsilon+1} \int_{\mathbb{R}} e^{\epsilon x}|\bar{U}|^{p^{*}+\epsilon+1} d x \\
= & I_{0}(\bar{U})-\frac{\beta}{p^{*}+1} \int_{\mathbb{R}}\left(e^{\epsilon x}-1\right)|\bar{U}|^{p^{*}+\epsilon+1} d x \\
& +\left(\frac{1}{p^{*}+1}-\frac{1}{p^{*}+1+\epsilon}\right) \beta \int_{\mathbb{R}} e^{\epsilon x}|\bar{U}|^{p^{*}+\epsilon+1} d x \\
& +\frac{\beta}{p^{*}+1} \int_{\mathbb{R}}\left(|\bar{U}|^{p^{*}+1}-|\bar{U}|^{p^{*}+\epsilon+1}\right) d x \\
= & I_{0}(\bar{U})-\frac{\beta}{p^{*}+1} \int_{\mathbb{R}}\left(e^{\epsilon x}-1\right)|\bar{U}|^{p^{*}+\epsilon+1}+A_{\epsilon} .
\end{aligned}
$$

where

$$
\begin{gathered}
A_{\epsilon}=\left(\frac{1}{p^{*}+1}-\frac{1}{p^{*}+1+\epsilon}\right) \beta \int_{\mathbb{R}} e^{\epsilon x}|\bar{U}|^{p^{*}+\epsilon+1} d x \\
+\frac{\beta}{p^{*}+1} \int_{\mathbb{R}}\left(|\bar{U}|^{p^{*}+1}-|\bar{U}|^{p^{*}+\epsilon+1}\right) d x
\end{gathered}
$$

As in [13], we can prove that

$$
A_{\epsilon}=k \epsilon \beta\left(\frac{1}{\left(1+p^{*}\right)^{2}} \int_{\mathbb{R}}|U|^{p^{*}+1} d x-\frac{1}{\left(1+p^{*}\right)} \int_{\mathbb{R}}|U|^{p^{*}+1} \log U d x\right)+o(\epsilon) .
$$

Also, by reasoning in a similar manner we have

$$
\begin{gathered}
\int_{\mathbb{R}}\left(e^{\epsilon x}-1\right)|\bar{U}|^{p^{*}+\epsilon+1} d x=\epsilon \int_{\mathbb{R}} x|\bar{U}|^{p^{*}+\epsilon+1} d x+o(\epsilon) \\
=\epsilon\left(\sum_{l=1}^{k} \xi_{l}\right) \int_{\mathbb{R}} U^{p^{*}+1} d y+o(\epsilon) .
\end{gathered}
$$

and

$$
I_{0}(\bar{U})=k I_{0}(U)-\beta C_{N} \int_{\mathbb{R}} U^{p^{*}} e^{x} d x\left(\sum_{l=2}^{k} e^{\xi_{l}-\xi_{l-1}}\right)+o(\epsilon)
$$


Now we need to evaluate $\int_{\mathbb{R}} \omega(x) e^{-\left(p^{*}-q\right) x}|\bar{U}|^{q+1} d x$. By following the argument in [11] et al and using our choice of $\xi_{l}^{\prime} s$, we have

$$
\begin{aligned}
\int_{\mathbb{R}} \omega(x) e^{-\left(p^{*}-q\right) x}|\bar{U}|^{q+1} d x & =\sum_{i=1}^{k} \int_{\mathbb{R}} \omega(x) e^{-\left(p^{*}-q\right) x}\left|U_{i}\right|^{q+1} d x+o(\epsilon) \\
& =\int_{\mathbb{R}} \omega(x) e^{-\left(p^{*}-q\right) x}\left|U_{1}\right|^{q+1} d x+o(\epsilon) .
\end{aligned}
$$

On the other hand, the following holds

$$
\begin{gathered}
\int_{\mathbb{R}} \omega(x) e^{-\left(p^{*}-q\right) x}\left|U_{1}\right|^{q+1} d x=e^{-\left(p^{*}-q\right) \xi_{1}} \int_{\mathbb{R}} \omega\left(x+\xi_{1}\right) e^{-\left(p^{*}-q\right) x}\left|U_{1}\left(x+\xi_{1}\right)\right|^{q+1} d x \\
=e^{-\left(p^{*}-q\right) \xi_{1}} \int_{\mathbb{R}} \omega\left(x+\xi_{1}\right) e^{-\left(p^{*}-q\right) x}|U|^{q+1} d x \\
=e^{-\left(p^{*}-q\right) \xi_{1}} V(0) \int_{\mathbb{R}} e^{-\left(p^{*}-q\right) x}|U|^{q+1} d x+o(1) .
\end{gathered}
$$

We thus have the following

$$
\begin{aligned}
E_{\epsilon}(\bar{U})= & I_{\epsilon}(\bar{U})+\frac{\beta}{q+1} \int_{\mathbb{R}} \omega(x) e^{-\left(p^{*}-q\right) x}|\bar{U}|^{q+1} d x \\
= & I_{0}(\bar{U})-\frac{\beta}{p^{*}+1} \int_{\mathbb{R}}\left(e^{\epsilon x}-1\right)|\bar{U}|^{p^{*}+\epsilon+1} d x+A_{\epsilon}+ \\
& +\frac{\beta}{q+1} \int_{\mathbb{R}} \omega(x) e^{-\left(p^{*}-q\right) x}|\bar{U}|^{q+1} d x \\
= & k I_{0}(U)-\beta C_{N} \int_{\mathbb{R}} U^{p^{*}} e^{x} d x\left(\sum_{l=2}^{k} e^{\xi_{l}-\xi_{l-1}}\right) \\
& -\frac{\beta}{p^{*}+1}\left(\epsilon\left(\sum_{l=1}^{k} \xi_{l}\right) \int_{\mathbb{R}} U^{p^{*}+1} d y\right) \\
& +k \epsilon \beta\left(\frac{1}{\left(1+p^{*}\right)^{2}} \int_{\mathbb{R}}|U|^{p^{*}+1} d x-\frac{1}{1+p^{*}} \int_{\mathbb{R}}|U|^{p^{*}+1} \log U d x\right)+ \\
& +\frac{\beta}{q+1}\left(e^{-\left(p^{*}-q\right) \xi_{1}} V(0) \int_{\mathbb{R}} e^{-\left(p^{*}-q\right) x}|U|^{q+1} d x\right)+o(\epsilon)
\end{aligned}
$$

which lead us to the following expression:

$$
E_{\epsilon}(\bar{U})=k a_{1}-a_{2} \sum_{l=2}^{k} e^{-\left(\xi_{l}-\xi_{l-1}\right)}-\epsilon a_{3}\left(\sum_{i=1}^{k} \xi_{i}\right)+k \epsilon \beta a_{4}+a_{5} V(0) e^{-\left(p^{*}-q\right) \xi_{1}}+o(\epsilon) .
$$


By using our choice of $\xi_{i}$ 's

$$
E_{\epsilon}(\bar{U})=k a_{1}+\epsilon \Psi_{k}(\Lambda)-\frac{a_{3} k}{2\left(p^{*}-q\right)}\left((1-k)\left(p^{*}-q\right)-2\right) \epsilon \log \epsilon+k \epsilon \beta a_{4} \epsilon+o(\epsilon),
$$

where $\Psi_{k}(\Lambda)$ is given by (18) and the constants $a_{i}, i=1, \cdots, 5$, are explicitly expressed as follows

$$
\left\{\begin{array}{l}
a_{1}=I_{0}(U), \quad a_{2}=\beta C_{N} \int_{\mathbb{R}} U^{p^{*}}(x) e^{x} d x, \quad a_{3}=\frac{\beta}{p^{*}+1} \int_{\mathbb{R}} U^{p^{*}+1}(x) d x \\
a_{4}=\frac{1}{\left(p^{*}+1\right)^{2}} \int_{\mathbb{R}} U^{p^{*}+1}(x) d x-\frac{1}{p^{*}+1} \int_{\mathbb{R}} U^{p^{*}+1}(x) \log U(x) d x \\
a_{5}=\frac{\beta}{q+1} \int_{\mathbb{R}} e^{-\left(p^{*}-q\right) x} U^{q+1}(x) d x
\end{array}\right.
$$

Notice that the term $o(\epsilon)$ in the above expression of $E_{\epsilon}(\bar{U})$ is uniform in the set of the $\Lambda_{i}^{\prime} s$ satisfying (17). A similar computation shows that differentiation with respect to the $\Lambda_{i}^{\prime} s$ leaves the term $o(\epsilon)$ of the same order in the $C^{1}$-sense.

\section{The finite dimensional reduction}

We consider again points $0<\xi_{1}<\xi_{2}<\cdots<\xi_{k}$ which are for now arbitrary and define

$$
Z_{i}(x)=U_{i}^{\prime}(x), \quad i=1, \cdots, k .
$$

Next we consider the problem of finding a function $\phi$ for which there are constants $c_{i}, i=1, \cdots, k$, such that, in $\mathbb{R}$

$$
\left\{\begin{array}{l}
\sum_{i=1}^{k} c_{i} Z_{i}=-(\bar{U}+\phi)^{\prime \prime}+(\bar{U}+\phi)-\beta\left[e^{\epsilon x}(\bar{U}+\phi)_{+}^{p^{*}+\epsilon}-\omega(x) e^{-\left(p^{*}-q\right) x}(\bar{U}+\phi)^{q}\right] \\
\phi(x) \rightarrow 0,|x| \rightarrow \infty, \\
\int_{\mathbb{R}} Z_{i} \phi d x=0, \quad i=1, \cdots, k
\end{array}\right.
$$

Let us consider the linearized operator around $\bar{U}$

$$
\mathcal{L}_{\epsilon} \phi=-\phi^{\prime \prime}+\phi-\beta\left[\left(p^{*}+\epsilon\right) e^{\epsilon x} \bar{U}^{p^{*}+\epsilon-1}-q \omega(x) e^{-\left(p^{*}-q\right) x} \bar{U}^{q-1}\right] \phi .
$$


Then (20) can be rewritten as

$$
\left\{\begin{array}{l}
\mathcal{L}_{\epsilon} \phi=N_{\epsilon}^{1}(\phi)+N_{\epsilon}^{2}(\phi)+R_{\epsilon}+\sum_{i=1}^{k} c_{i} Z_{i} \\
\phi(x) \rightarrow 0,|x| \rightarrow \infty \\
\int_{\mathbb{R}} Z_{i} \phi d x=0, \quad i=1, \cdots, k
\end{array}\right.
$$

where

$$
\begin{gathered}
N_{\epsilon}^{1}=\beta e^{\epsilon x}\left[(\bar{U}+\phi)^{p^{*}+\epsilon}-\bar{U}^{p^{*}+\epsilon}-\left(p^{*}+\epsilon\right) \bar{U}^{p^{*}+\epsilon-1} \phi\right], \\
N_{\epsilon}^{2}=-\beta \omega(x) e^{-\left(p^{*}-q\right) x}\left[(\bar{U}+\phi)^{q}-\bar{U}^{q}-q \bar{U}^{q-1} \phi\right], \\
R_{\epsilon}=\sum_{i=1}^{k} U_{i}^{p^{*}}+\beta e^{\epsilon x} \bar{U}^{p^{*}+\epsilon}-\beta \omega(x) e^{-\left(p^{*}-q\right) x} \bar{U}^{q} .
\end{gathered}
$$

Next we prove that (21) has a solution for certain choice of $\xi_{i}$. In order to do that we first analyze its linear part, i.e., we consider the problem of, given a function $h$, finding $\phi$ such that

$$
\left\{\begin{array}{l}
\mathcal{L}_{\epsilon} \phi=h+\sum_{i=1}^{k} c_{i} Z_{i} \\
\phi(x) \rightarrow 0,|x| \rightarrow \infty, \\
\int_{\mathbb{R}} Z_{i} \phi d x=0, \quad i=1, \cdots, k
\end{array}\right.
$$

In order to analyze invertibility properties of $\mathcal{L}_{\epsilon}$ under the orthogonality conditions, we introduce the following norm for function $\psi: \mathbb{R} \rightarrow \mathbb{R}$

$$
\|\psi\|_{*}=\sup _{x \in \mathbb{R}}\left(\sum_{i=1}^{k} e^{-\sigma|x-\xi|}\right)^{-1}|\psi(x)|
$$

where $\sigma>0$ is a small constant to be fixed later.

The following result holds.

Proposition 3.1. There exist positive numbers $\epsilon_{0}, \delta_{0}, R_{0}$ such that if

$$
R_{0}<\xi_{i}, \quad R_{0}<\min _{1 \leq i<k}\left(\xi_{i+1}-\xi_{i}\right), \quad \xi_{k}<\frac{\delta_{0}}{\epsilon}
$$


then for all $0<\epsilon<\epsilon_{0}$ and for all $h \in C(\mathbb{R})$ with $|h|_{*}<+\infty$, problem (22) has a unique solution $\psi=: T_{\epsilon}(h)$, such that

$$
\left\|T_{\epsilon}(h)\right\|_{*} \leq C\|h\|_{*}, \quad\left|c_{i}\right| \leq\|h\|_{*} .
$$

Lemma 3.1. Assume there is a sequence $\epsilon_{n} \rightarrow 0$ and points $\xi_{i}$ 's satisfying $0<$ $\xi_{1}^{n}<\cdots<\xi_{k}^{n}$ with

$$
\xi_{1}^{n} \rightarrow \infty, \quad \min _{1 \leq i<k}\left(\xi_{i+1}^{n}-\xi_{i}^{n}\right) \rightarrow \infty, \quad \xi_{k}^{n}=o\left(\epsilon_{n}^{-1}\right)
$$

such that for certain functions $\phi_{n}$ and $h_{n}$ with $\left\|h_{n}\right\|_{*} \rightarrow 0$, and scalars $c_{i}^{n}$, one has in $\mathbb{R}$

$$
\left\{\begin{array}{l}
\mathcal{L}_{\epsilon_{n}}\left(\phi_{n}\right)=h_{n}+\sum_{i=1}^{k} c_{i}^{n} Z_{i}^{n} \\
\phi_{n}(x) \rightarrow 0,|x| \rightarrow \infty, \\
\int_{\mathbb{R}} Z_{i}^{n} \phi_{n} d x=0, \quad i=1, \cdots, k
\end{array}\right.
$$

with $Z_{i}^{n}(x)=U^{\prime}\left(x-\xi_{i}^{n}\right)$. Then $\lim _{n \rightarrow \infty}\left\|\phi_{n}\right\|_{*}=0$

Proof. We first establish the weaker assertion that

$$
\lim _{n \rightarrow \infty}\left\|\phi_{n}\right\|_{\infty}=0
$$

By contradiction, we may assume that $\left\|\phi_{n}\right\|_{\infty}=1$. Testing (25) against $Z_{l}^{n}$ and integrating by parts we get

$$
\sum_{i=1}^{k} c_{i}^{n} \int_{\mathbb{R}} Z_{i}^{n} Z_{l}^{n} d x=\int_{\mathbb{R}} \mathcal{L}_{\epsilon_{n}}\left(Z_{l}^{n}\right) \phi_{n} d x-\int_{\mathbb{R}} h_{n} Z_{l}^{n} d x .
$$

This defines a linear system in the $c_{i}$ 's which is "almost diagonal" as $n \rightarrow \infty$. Moreover, the assumptions made plus the fact that the $Z_{l}^{n}$ solves

$$
-Z^{\prime \prime}+\left(1-p^{*} \beta U_{l}^{p^{*}-1} Z\right)=0
$$

yields, after an application of dominated convergence, that $\lim _{n \rightarrow \infty} c_{i}^{n}=0$. If we set $x_{n} \in \mathbb{R}^{N}$ such that $\phi_{n}\left(x_{n}\right)=1$, we can assume that there exists $i \in\{1, \cdots, k\}$ such that for $n$ large enough such that $\left|\xi_{l}^{n}-x_{n}\right|<R$ for some fixed $R>0$. We set 
$\tilde{\phi}_{n}=\phi_{n}\left(x+\xi_{i}^{n}\right)$. From (25), we see that passing to a suitable subsequence, $\tilde{\phi}_{n}(x)$ converges uniformly over compacts to a nontrivial bounded solution $\tilde{\phi}$ of

$$
-\tilde{\phi}^{\prime \prime}+\tilde{\phi}-\beta p^{*} U^{p^{*}} \tilde{\phi}=0, \quad \text { in } \mathbb{R} .
$$

Hence for some $c \neq 0, \tilde{\phi}=c U^{\prime}$. However the orthogonality condition passes to the limit as

$$
0=\int_{\mathbb{R}} Z_{l}^{n} \phi_{n} \rightarrow c \int \mathbb{R}\left(U^{\prime}\right)^{2}
$$

which is a contradiction. Then $\lim _{n \rightarrow \infty}\left\|\phi_{n}\right\|_{\infty}=0$.

Now, we observe that shows that (25) takes the form

$$
-\phi_{n}^{\prime \prime}+\phi_{n}=g_{n}
$$

where

$$
g_{n}=h_{n}+\sum_{i=1}^{k} c_{i}^{n} Z_{i}^{n}+\beta\left[\left(p^{*}+\epsilon_{n}\right) e^{\epsilon_{n} x} \bar{U}^{p^{*}+\epsilon_{n}-1}-q \omega(x) e^{-\left(p^{*}-q\right) x} \bar{U}^{q-1}\right] \phi_{n} .
$$

We estimate $g_{n}$,

$$
\begin{aligned}
\left|g_{n}\right| \leq & \left\|h_{n}\right\|_{*}\left(\sum_{i=1}^{k} e^{-\sigma\left|x-\xi_{i}^{n}\right|}\right)+c_{l}^{n} \sum_{i=1}^{n} o\left(e^{-\left|x-\xi_{i}^{n}\right|}\right)+ \\
& +\left\|\phi_{n}\right\|_{\infty}\left(\sum_{i=1}^{n} o\left(e^{-\left(p^{*}-1\right)\left|x-\xi_{i}^{n}\right|}\right)+\sum_{i=1}^{n} o\left(e^{-\left(2 q-p^{*}-1\right)\left|x-\xi_{i}^{n}\right|}\right)\right),
\end{aligned}
$$

since $V \in L^{\infty}(\mathbb{R})$. If $0<\sigma<\min \left\{1, p^{*}-1,2 q-p^{*}-1\right\}$, we have

$$
\left|g_{n}(x)\right| \leq \theta_{n} \sum_{i=1}^{k} e^{-\sigma\left|x-\xi_{i}\right|}=: \psi_{n}(x),
$$

with $\theta_{n} \rightarrow 0$. We see the that the function $C \psi_{n}$, for $C>0$ sufficiently large, is a supersolution for (26), so that $\phi_{n} \leq C \psi_{n}$. Similarly, we have $\phi_{n} \geq-C \psi_{n}$. Thus, the proof is concluded.

Then the proof of Proposition 3.1 follows from Lemma 3.1] as in [11].

Next we study some differentiability properties of $T_{\epsilon}$ on $\xi_{i}$. We write $\xi=$ $\left(\xi_{1}, \cdots, \xi_{k}\right)$. We let $\mathcal{C}_{*}$ be the Banach space of all continuous $\psi$ defined in $\mathbb{R}$ satisfying $\|\psi\|_{*}<\infty$, endowed with the norm $\|\cdot\|_{*}$. Also, let $\mathcal{L}\left(C_{*}\right)$ be the space of linear operators of $C_{*}$.

The following result can be established. 
Proposition 3.2. Under the assumptions of the Proposition 3.1 consider the map $T_{\epsilon}(\xi)$ with values in $\mathcal{L}\left(C_{*}\right)$. Then $T_{\epsilon}$ is $C^{1}$ and

$$
\left\|D_{\xi} T_{\xi}\right\|_{\mathcal{L}\left(C_{*}\right)} \leq C
$$

uniformly on $\xi$ satisfying (23), for some constant $C$.

Proof. Fix $h \in C_{*}$ and let $\phi=T_{\epsilon}(h)$ for $\epsilon<\epsilon_{0}$. Notice that $\phi$ satisfies (22) and the orthogonality conditions, for some constants $c_{i}$ uniquely determined. For $l \in\{1, \cdots, k\}$, if we define the constant $b_{l}$ as follows

$$
b_{l} \int_{\mathbb{R}}\left|Z_{l}\right|^{2}=\int_{\mathbb{R}} \phi \partial_{\xi_{l}} Z_{l} \text {. }
$$

By differentiating with respect to $\xi_{l}$ we obtain that

$$
\partial_{\xi_{l}} \phi=T_{\epsilon}(f)+b_{l} Z_{l}
$$

where

$$
f=-b_{l} \mathcal{L}_{\epsilon} Z_{l}+c_{l} \partial_{\xi_{l}} Z_{l}+\beta\left[\left(p^{*}+\epsilon\right) e^{\epsilon x}\left(\partial_{\xi_{l}} \bar{U}^{p^{*}+\epsilon-1}\right)-q \omega(x) e^{-\left(p^{*}-q\right) x}\left(\partial_{\xi_{l}} \bar{U}^{q-1}\right)\right] \phi .
$$

Moreover $\|f\|_{*} \leq C\|h\|_{*},\left|b_{l}\right| \leq C\|\phi\|_{*}$ so that $\left\|\partial_{\xi_{l}} \phi\right\| \leq C\|h\|_{*}$. Besides $\partial_{\xi_{l}} \phi$ depends continuously on $\xi$ for this norm. Thus, the result follows.

We are now ready to prove that (21) is uniquely solvable with respect to $\|\phi\|_{*}$. In order to do that we restrict the range of the parameters $\xi_{i}$ 's in a convenient way. We assume that, for a fixed $M>0$ large, the following conditions hold

$$
\log (M \epsilon)^{-1}<\min _{1 \leq i<k}\left(\xi_{i+1}-\xi_{i}\right), \quad \xi_{k}<k \log (M \epsilon)^{-1} .
$$

Then we can estimate $R_{\epsilon}, N_{\epsilon}^{1}+N_{\epsilon}^{2}$, and their derivatives, by direct calculation, as follows.

Lemma 3.2. If $\|\phi\|_{1} \leq \frac{1}{2}\|\bar{U}\|_{1}$ then

$$
\begin{aligned}
& \left\|N_{\epsilon}(\phi)\right\|_{*} \leq C\left(\|\phi\|_{*}^{\min \left\{p^{*}, 2\right\}}+\|\phi\|_{*}^{\min \left\{2 q-p^{*}, 2\right\}}\right) \\
& \left\|D_{\phi} N_{\epsilon}(\phi)\right\|_{*} \leq C\left(\|\phi\|_{*}^{\min \left\{p^{*}-1,2\right\}}+\|\phi\|_{*}^{\min \left\{2 q-p^{*}-1,2\right\}}\right)
\end{aligned}
$$

where $\|\phi\|_{1}:=\sup _{x \in \mathbb{R}}\left(\sum_{i=1}^{k} e^{\left|x-\xi_{i}\right|}\right)^{-1}|\phi(x)|$ and $N_{\epsilon}(\phi)=N_{\epsilon}^{1}(\phi)+N_{\epsilon}^{2}(\phi)$. In addition, if (27) holds then

$$
\left\|R_{\epsilon}\right\|_{*} \leq C \epsilon^{\frac{1+\tau}{2}}, \quad\left\|\partial_{\xi} R_{\epsilon}\right\|_{*} \leq C \epsilon^{\frac{1+\tau}{2}}
$$

where $\tau>0$ is small. 
The next result allows for the reduction to a finite dimensional problem, as we will see in the next section. The proof is very similar to [11, Proposition 3] and we omit it here.

Proposition 3.3. Assume (27) holds. Then, for all $\epsilon$ small enough, there exists a unique solution $\phi=\phi(\xi)$ to problem (20), which satisfies

$$
\|\phi\|_{*} \leq C \epsilon^{\frac{1+\tau}{2}}
$$

Moreover, the map $\xi \mapsto \phi(\xi)$ is of class $C^{1}$ for the norm $\|\cdot\|_{*}$ and

$$
\left\|D_{\xi} \phi\right\|_{*} \leq C \epsilon^{\frac{1+\tau}{2}}
$$

\section{The finite dimensional variational problem}

In this section we fix a large constant $M>0$ and assume the conditions (27) for $\xi$. Our problem is equivalent to that of finding $\xi_{i}$ 's satisfying $c_{i}(\xi)=0$, for all $i=1,2, \cdots, k$. In this case, $v=\bar{U}+\phi$ is a solution for (10) satisfying the desired formula.

We consider the functional

$$
\mathcal{I}_{\epsilon}(\xi)=E_{\epsilon}(\bar{U}+\phi)
$$

where $\phi=\phi(\xi)$ is that of Proposition 3.3 and $E_{\epsilon}$ is the energy functional defined in (11). It is known that finding the desired $c_{i}$ 's is equivalent to finding a critical point of $\mathcal{I}_{\epsilon}(\xi)$, see for instance [11]. That is, we need to find a point $\xi$ satisfying

$$
\nabla \mathcal{I}_{\epsilon}(\xi)=0
$$

In order to do that, the following expansion result will be crucial.

Lemma 4.1. The following expansion holds

$$
\mathcal{I}_{\epsilon}(\xi)=E_{\epsilon}(\bar{U})+o(\epsilon)
$$

where $o(\epsilon)$ is uniform in the $C^{1}$-sense over all points $\xi$ satisfying (27). 
Proof. First, notice that $D E_{\epsilon}(\bar{U}+\phi)[\phi]=0$. It then follows from Taylor expansion that

$$
\begin{aligned}
E_{\epsilon}(\bar{U}+\phi)-E_{\epsilon}(\bar{U}) & =\int_{0}^{1} D^{2} E_{\epsilon}(\bar{U}+t \phi)\left[\phi^{2}\right] t d t \\
& =\int_{0}^{1} \int_{\mathbb{R}}\left[N_{\epsilon}(\phi)+R_{\epsilon}\right] \phi t d t \\
& +\int_{0}^{1} \int_{\mathbb{R}} \beta\left(p^{*}+\epsilon\right) e^{\epsilon x}\left[\bar{U}^{p^{*}+\epsilon-1}-(\bar{U}+t \phi)^{p^{*}+\epsilon-1}\right] \phi^{2} t d t \\
& -\int_{0}^{1} \beta q \int_{\mathbb{R}} \omega(x) e^{-\left(p^{*}-q\right) x}\left[\bar{U}^{q-1}-(\bar{U}+t \phi)^{q-1}\right] \phi^{2} t d t
\end{aligned}
$$

Since $\|\phi\|_{*} \leq C e^{\frac{1+\tau}{2}}$, from Lemma 3.2, we get

$$
\mathcal{I}_{\epsilon}(\xi)-E_{\epsilon}(\bar{U})=o\left(e^{1+\tau}\right)
$$

uniformly on points satisfying (27). Next we differentiate with respect to $\xi$ and get, from (30) that

$$
\begin{aligned}
D_{\xi}\left[\mathcal{I}_{\epsilon}(\xi)-E_{\epsilon}(\bar{U})\right] & =\int_{0}^{1} \int_{\mathbb{R}} D_{\xi}\left[N_{\epsilon}(\phi)+R_{\epsilon}\right] \phi t d t \\
& +\int_{0}^{1} \int_{\mathbb{R}} \beta\left(p^{*}+\epsilon\right) e^{\epsilon x} D_{\xi}\left[\bar{U}^{p^{*}+\epsilon-1}-(\bar{U}+t \phi)^{p^{*}+\epsilon-1}\right] \phi^{2} t d t \\
& -\int_{0}^{1} \beta q \int_{\mathbb{R}} \omega(x) e^{-\left(p^{*}-q\right) x} D_{\xi}\left[\bar{U}^{q-1}-(\bar{U}+t \phi)^{q-1}\right] \phi^{2} t d t .
\end{aligned}
$$

Using similar arguments as in Proposition 3.2, we find that

$$
D_{\xi}\left[\mathcal{I}_{\epsilon}(\xi)-E_{\epsilon}(\bar{U})\right]=o\left(\epsilon^{1+\tau}\right)
$$

Thus the result follows.

In what follows we prove Theorem 1.1

Proof of Theorem 1.1 Recall that

$$
\begin{gathered}
\xi_{1}=-\frac{1}{p^{*}-q} \log \epsilon-\log \Lambda_{1} \\
\xi_{i+1}-\xi_{i}=-\log \epsilon-\log \Lambda_{i+1}, \quad i=1, \cdots, k-1
\end{gathered}
$$


where $\Lambda_{i}$ 's are positive parameters. Thus, it is sufficient to find a critical point of

$$
\Phi_{\epsilon}(\Lambda)=\epsilon^{-1} \mathcal{I}_{\epsilon}(\xi(\Lambda))
$$

Now, from Lemma 3.1, we get

$$
\nabla \Phi_{\epsilon}(\Lambda)=\nabla \Psi_{k}+o(1)
$$

where $o(1)$ is uniform with respect to parameters $\Lambda$ with $M^{-1}<\Lambda_{i}<M$, for fixed large $M$.

Next we analyze the critical points of $\Psi_{k}(\Lambda)$, by writing

$$
\Psi_{k}(\Lambda)=\varphi_{1}\left(\Lambda_{1}\right)+\sum_{i=2}^{k} \varphi_{i}\left(\Lambda_{i}\right)
$$

where

$$
\begin{aligned}
\varphi_{k}(s) & =a_{5} V(0) s^{p^{*}-q}+a_{3} k \log s, \\
\varphi_{i}(s) & =(k-i+1) a_{3} \log s-a_{2} s, \quad i=2, \cdots, k .
\end{aligned}
$$

Notice that, $\varphi_{i}$ has a unique maximum point $\Lambda_{i}^{*}=(k-i+1) \frac{a_{3}}{a_{2}}$, for $i=2, \cdots, k$. If we further assume that $V(0)<0$, then $\varphi_{1}(s)$ also has a unique maximum point $\Lambda_{1}^{*}=\left[-\frac{a_{3} k}{a_{5} V(0)\left(p^{*}-q\right)}\right]^{\frac{1}{p^{*}-q}}$.

Since the critical point

$$
\Lambda^{*}=\left(\left[-\frac{a_{3} k}{a_{5} V(0)\left(p^{*}-q\right)}\right]^{\frac{1}{p^{*}-q}}, \frac{(k-1) a_{3}}{a_{2}}, \cdots, \frac{a_{3}}{a_{2}}\right)
$$

of $\Psi_{k}$ is nondegenerate, it follows that the local degree $\operatorname{deg}\left(\nabla \Psi_{k}, \mathcal{V}, 0\right)$ is well defined and nonzero. Here $\mathcal{V}$ denotes a small neighborhood of $\Lambda^{*}$ in $\mathbb{R}^{k}$. Hence, $\operatorname{deg}\left(\nabla \Phi_{\epsilon}, \mathcal{V}, 0\right) \neq 0$, if $\epsilon$ is small enough. We conclude that there exists a critical point $\Lambda_{\epsilon}^{*}$ of $\Phi_{\epsilon}$ satisfying

$$
\Lambda_{\epsilon}^{*}=\Lambda^{*}+o(1)
$$

For $\xi_{\epsilon}=\xi\left(\Lambda_{\epsilon}^{*}\right)$, the functions

$$
v=\bar{U}+\phi\left(\xi_{\epsilon}\right)
$$

are solutions of (10). From the equation (20) and Proposition 3.3, we derive that $v=\bar{U}(1+o(1))$. If we set $\xi^{*}=\xi\left(\Lambda^{*}\right)$, then it is also true that

$$
v(x)=\sum_{i=1}^{k} U\left(x-\xi_{i}^{*}\right)(1+o(1)) .
$$


Now, changing the variables back, we have that

$$
u_{\epsilon}^{*}(r)=\gamma_{N} \sum_{i=1}^{k}\left(\frac{1}{1+e^{\left(p^{*}-1\right) \xi_{i}^{*}} r^{2}}\right)^{\frac{N-2}{2}} e^{\xi_{i}^{*}}(1+o(1)),
$$

where $e^{\xi_{i}^{*}}=e^{-(i-1)-\frac{1}{p^{*}-q}} \prod_{j=1}^{i}\left(\Lambda_{j}^{*}\right)^{-1}$, is a solution of $(8)$. We conclude that the ansatz given for $v$ provides a spike-tower solution for (2).

\section{Proof of Theorem 1.2}

In this section we prove Theorem 1.2. The proof is very similar to that of Theorem 1.1. then we just highlight below the most critical changes. Since we are seeking for radial solutions of (1), we consider again the following slightly supercritical equation

$$
\left\{\begin{array}{l}
u^{\prime \prime}(r)+\frac{N-1}{r} u^{\prime}(r)+u^{p^{*}+\epsilon}(r)-V(r) u^{q}(r)=0 \\
u(r) \rightarrow 0, \text { as } r \rightarrow \infty
\end{array}\right.
$$

with $\epsilon>0$, but this tame we take $q>p^{*}$. We consider the transformation

$$
v(x)=r^{\frac{2}{p^{*}-1}} u(r), \quad \text { with } r=e^{\frac{p^{*}-1}{2} x},
$$

for $x \in \mathbb{R}$. Then the problem (30) becomes

$$
\left\{\begin{array}{l}
v^{\prime \prime}(x)-v(x)+\beta\left[e^{-\epsilon x} v^{p^{*}+\epsilon}(x)-V\left(e^{\frac{p^{*}-1}{2} x}\right) e^{\left(p^{*}-q\right) x} v^{q}(x)\right]=0, \\
0<v(x) \rightarrow 0, \text { as }|x| \rightarrow \infty
\end{array}\right.
$$

We recall that $\beta=\left(\frac{2}{N-2}\right)^{2}$. Again we denote $\omega(x)=V\left(e^{\frac{p^{*}-1}{2} x}\right)$.

The energy functional related to (32) is

$$
\hat{E}_{\epsilon}(\psi)=\hat{I}_{\epsilon}(\psi)+\frac{\beta}{q+1} \int_{\mathbb{R}} \omega(x) e^{\left(p^{*}-q\right) x}|\psi|^{q+1} d x
$$

where

$$
\hat{I}_{\epsilon}(\psi)=\frac{1}{2} \int_{\mathbb{R}}\left(\left|\psi^{\prime}\right|^{2}+|\psi|^{2}\right) d x-\frac{\beta}{p^{*}+\epsilon+1} \int_{\mathbb{R}} e^{-\epsilon x}|\psi|^{p^{*}+\epsilon+1} d x .
$$


We choose, for small $\epsilon>0$, the points $\xi_{i}$ as follows:

$$
\begin{gathered}
\hat{\xi}_{1}=-\frac{1}{q-p^{*}} \log \epsilon-\log \hat{\Lambda}_{1} \\
\hat{\xi}_{i+1}-\hat{\xi}_{i}=-\log \epsilon-\log \hat{\Lambda}_{i+1}, \quad i=1, \cdots, k-1
\end{gathered}
$$

where the $\hat{\Lambda}_{i}$ 's are positive parameters. We want to find a solution of (32) of the form

$$
\hat{v}(x)=\sum_{i=1}^{k} U\left(x-\hat{\xi}_{i}\right)+\phi(x)
$$

where $U$ is defined by (16) and $\phi$ is small. We set $\hat{\Lambda}=\left(\hat{\Lambda}_{1}, \hat{\Lambda}_{2}, \cdots, \hat{\Lambda}_{k}\right)$.

In this setting, Lemma 3.1 takes the following form.

Lemma 5.1. Let $N \geq 3, \delta>0$ fixed, $k \in \mathbb{N}$. Assume that

$$
\delta<\hat{\Lambda}_{i}<\delta^{-1}, \quad i=1,2, \cdots, k .
$$

Then there exist positive numbers $a_{1}, i=1, \cdots, 4$ and $\hat{a}_{5}$, depending on $N, p$ and q, such that

$$
\hat{E}_{\epsilon}\left(\hat{U}_{S}\right)=k a_{1}+\epsilon \hat{\Psi}_{k}(\hat{\Lambda})+k \epsilon \beta a_{4}+\epsilon \hat{\theta}_{\epsilon}(\hat{\Lambda})-\frac{a_{3} k}{2\left(q-p^{*}\right)}\left((1-k)\left(q-p^{*}\right)-2\right) \epsilon \log \epsilon
$$

where

$$
\hat{\Psi}_{k}(\hat{\Lambda})=a_{3} k \log \hat{\Lambda}_{1}+\hat{a}_{5} V_{\infty} \Lambda_{1}^{\left(q-p^{*}\right)}+\sum_{i=1}^{k}\left[(k-i+1) a_{3} \log \hat{\Lambda}_{i}-a_{2} \hat{\Lambda}_{i}\right]
$$

and $\hat{\theta}_{\epsilon}(\hat{\Lambda}) \rightarrow 0$ as $\epsilon \rightarrow 0$ uniformly in $C^{1}$-sense on the set of $\hat{\Lambda}_{i}$ 's satisfying (35). Moreover, the constants $a_{i}, i=1,2, \cdots, 4$ are given as in (19) and $\hat{a}_{5}$ is defined by

$$
\hat{a}_{5}=\frac{\beta}{q+1} \int_{\mathbb{R}} e^{-\left(q-p^{*}\right) x} U^{q+1}(x) d x .
$$

If we assume that $V_{\infty}<0$ then $\hat{\Psi}_{k}$ has a unique nondegenerate critical point, given by

$$
\hat{\Lambda}^{*}=\left(\left[\frac{a_{3} k}{\left(p^{*}-q\right) \hat{a}_{5} V_{\infty}}\right]^{\frac{1}{q-p^{*}}}, \frac{(k-1) a_{3}}{a_{2}}, \frac{(k-2) a_{3}}{a_{2}}, \cdots, \frac{a_{3}}{a_{2}}\right) .
$$

The finite-dimensional reduction and the conclusion of the Theorem follows in a similar way to the proof of Theorem 1.1 . 


\section{Acknowledgements}

The first author is supported by FONDECYT Grant 1160135 and Millennium Nucleus Center for Analysis of PDE, NC130017. The second author was supported by FAPESP (Brazil) Grant \#2016/04925-7.

\section{References}

[1] T. Aubin. Problèmes isopérimétriques et espaces de Sobolev. J. Differential Geom. 11 (1976), 573-598.

[2] R. Bamón, M. del Pino and I. Flores. Ground states of semilinear elliptic equations: A geometric approach. Ann. Inst. H. Poincaré 17 (2000), 551581.

[3] V. Benci and G. Cerami. Existence of positive solutions of the equation $-\Delta u+a(x) u=u^{(N+2) /(N-2)}$ in $\mathbb{R}^{n}$. J. Funct. Anal. 88 (1990), 91-117.

[4] L. A. Caffarelli, B. Gidas and J. Spruck. Asymptotic symmetry and local behavior of semilinear elliptic equations involving critical Sobolev growth. Comm. Pure Appl. Math. 42 (1989), 271-297.

[5] C. C. Chen and C. -S. Lin. Blowing up with infinite energy of conformal metrics on $S^{n}$. Comm. Partial Differential Equations 24 (1999), 785-799.

[6] A. Contreras and M. del Pino. Nodal Bubble-Tower solutions to radial elliptic problems near criticality. Discrete Contin. Dyn. Syst. 16 (2006), 525-539.

[7] J. Davila and I. Guerra. Slowly decaying radial solutions of an elliptic equations with subcritical and supercritical exponents. J. D'Analyse Math. 129 (2016), 367-391.

[8] W. Y. Ding and W. M. Ni. On the existence of positive entire solutions of a semilinear elliptic equation. Arch. Rat. Mech. Anal. 91 (1986), 283-308.

[9] R. H. Fowler. Further studies on Emden's and similar differential equations. Quart. J. Math. 2 (1931), 259-288.

[10] J. Campos. "Bubble-Tower" phenomena in a semilinear elliptic equation with mixed Sobolev growth. Nonlinear Analysis. 68 (2008), 1382-1397. 
[11] A. M. Micheletti, M. Musso and A. Pistoia. Super-position of spikes for a slightly super-critical elliptic equation in $\mathbb{R}^{n}$. Discrete and Cont. Dyn. Syst. 12 (2005), 747-760.

[12] A. M. Micheletti and A. Pistoia. Existence of blowing-up solutions for a slightly sub-critical or a slightly supercritical non-linear elliptic equation on $\mathbb{R}^{n}$. Nonlinear Analysis T.M.A. 52 (2003), 173-195.

[13] M. del Pino, J. Dolbeault and M. Musso. "Bubble-Tower" radial solutions in the slightly super critical Brezis-Nirenberg problem. J. Differential Equations 193 (2003), 280-306.

[14] M. del Pino, M. Musso and A. Pistoia. Super critical boundary bubbling in a semilinear Neumann problem. Ann. Inst. H. Poincaré 22 (2005), 45-82.

[15] A. Floer and A. Weinstein. Nonspreading wave packets for the cubic Schrodinger equation with bounded potential. J. Funct. Anal. 69 (1986), 397-408.

[16] G. Talenti. Best constant in Sobolev inequality. Ann. Mat. Pura Appl. 110 (1976), 353-372. 